\title{
Neural Correlates of Visual-Motion Perception as Object- or Self-motion
}

\author{
Andreas Kleinschmidt,* Kai V. Thilo,† Christian Büchel,‡ Michael A. Gresty,§ \\ Adolfo M. Bronstein,§ and Richard S. J. Frackowiak $\emptyset$
}

\begin{abstract}
*Department of Neurology, J ohann Wolfgang GoetheUniversity, D-60590 Frankfurt, Germany; †Laboratory of Physiology, Oxford University, Oxford, United Kingdom; ‡Department of Neurology, Hamburg University, D-20246 Hamburg, Germany; §Academic Department of Neuro-otology, Division of Neuroscience and Psychological Medicine, Imperial College, London, United Kingdom; and IWell come Department of I maging Neuroscience, University College London, London, United Kingdom
\end{abstract}

Received November 30, 2001

Both self-motion and objects moving in our visual field generate visual motion by displacing images on the retina. Resolving this ambiguity may seem effortless but large-field visual-motion stimuli can yield perceptual rivalry between the real percept of objectmotion and the illusory percept of self-motion (vection). We used functional magnetic resonance imaging to record brain activity in human observers exposed to constant-velocity roll-motion. This stimulus induced responses in areas reaching from calcarine to parieto-occipital and to ventral and lateral temporo-occipital cortex and the anterior insula. During vection, early motion-sensitive visual areas and vestibular parieto-insular cortex deactivated, whereas higher-order parieto- and temporo-occipital areas known to respond to optical flow retained identical activity levels. Within this sustained response, these latter areas displayed transient activations in response to each perceptual switch as identified in event-related analyses. Our results thus show that these areas are responsive to the type of visual motion stimulus and highly sensitive to its perceptual bistability. The only region to be more active during perceived self-motion was in, or close to, the cerebellar nodulus. This activation may correspond to the gain increase of torsional optokinetic nystagmus during vection and/or to changes in sensory processing related to the rotational percept. In conclusion, we identified neural correlates of perceiving self-motion from vision alone, i.e., in the absence of confirmatory vestibular or proprioceptive input. These functional properties preserve the organism's ability to move accurately in its environment by relying on visual cues under conditions when the other spatial senses fail to provide such information. 2002 Elsevier Science (USA)

Key Words: visual system; circular vection; ego-motion; vestibular cortex; perceptual ambiguity; visual awareness; functional magnetic resonance imaging.

\section{INTRODUCTION}

Visual motion processing serves several perceptual purposes (Movshon et al., 1985; Nakayama, 1985; Albright and Stoner, 1995). It is one of the most powerful cues for binding objects ("form-from-motion"). Speedand direction-sensitive motion detectors covering the visual field will respond in synergy to object-motion, thus defining an object form, e.g., by coherence of displacement that its parts display against a background. Wide visual field coverage by the object and/or impoverished information from the stationary background, however, also permit a different perceptual judgement, namely that retinal shifting of an object is caused by observer motion (Mach, 1875; Helmholtz, 1896; Gibson, 1954; Warren, 1995; Lappe et al., 1999). While visual-motion reflects only relative motion information between object and observer, additional cues usually help to disambiguate object- from self-motion. Vestibular and proprioceptive sensory feedback and efference copies of body, head, and eye movements can prevent us from mistaking the origin of visual-motion (Wertheim, 1994; Wexler et al., 2001). However, when visual-motion is the sole informative cue for reconstructing self-motion a perceptual ambiguity may arise and induce the intermittent illusion of self-motion (Dichgans and Brandt, 1978), e.g., when a sensation of self-motion (vection) is evoked by a train slowly moving on a neighboring track as we watch from a stationary wagon.

To identify brain systems processing self-motion we chose the approach of using one constant visual-motion stimulus that was made sufficiently ambiguous to yield both percepts, i.e., object- and self-motion, in spontaneous alternation. With functional magnetic resonance imaging we recorded brain activity in human subjects who, exposed to this stimulus, fluctuated between perceiving a rotating object and feeling themselves rotate around the nearly earth-vertical axis of gaze while 
looking at an apparently stationary object (circular vection; see Fig. 1 and Materials and Methods). Thus, we addressed the distinction of object- and self-motion at the level of perceptual awareness rather than that of specialized visual feature processing. Any brain activity change correlating with either of the percepts cannot be accounted for by the stimulus properties per se. This avoids the confound of previous studies that generated illusory self-motion but compared related brain activity with conditions differing in physical stimulus properties, e.g., coherence of speed or direction (de J ong et al., 1994; Brandt et al., 1998).

\section{MATERIALS AND METHODS}

\section{Functional Imaging}

Data from eight healthy subjects (written informed consent, one female, seven males, age 22-48 years) were acquired on a $2 \mathrm{~T}$ magnetic resonance imager (Siemens Vision, Erlangen, Germany; head coil), obtaining a structural (T1-weighted) scan and then series of blood-oxygenation-sensitive (T2*-weighted) echoplanar image volumes every $4.1 \mathrm{~s}$ (image repetition time/ echo time $=80.7 / 40 \mathrm{~ms} ; 48$ contiguous transverse slices, voxel size $=3 \times 3 \times 3 \mathrm{~mm}^{3}$ ).

\section{Visual Stimulation}

Prior to each scanning series (118 image volumes, 8 min duration, three runs), subjects started looking at the rotational center of a disk with a windmill pattern (12 fluorescent radial stripes alternating with black stripes of equivalent width subtending approximately radially $45^{\circ}$ of the visual field) in an otherwise dimmed scanner room. The disk was mounted above the head coil (approximately $12 \mathrm{~cm}$ viewing distance to center) and mechanically controlled by a cogwheel connection to a propylene rod that was driven by a motor in the console room. For the first 18 image volumes the disk remained stationary and then started rotating about its center at a constant speed $\left(45^{\circ} / \mathrm{s}\right)$. We used both clockwise and counter-clockwise rotation in different sessions. Since no significant brain activity differences were found between these conditions, the data were pooled for the other analyses. Previous measurements with an almost identical stimulus under video-oculography (Thilo et al., 1999) established that perception is bistable and that subjects can maintain fixation over the length of time chosen for the runs.

\section{Bistable Perception}

Key presses with the right index finger indicated the onset of visual-motion stimulation and of epochs with perceived object-motion. Middle finger key presses indicated the onset of illusory self-motion, i.e., circular vection. Thus, a sequence of alternating key presses was recorded, defining the subjects' bistable perception of the stimulus (see Fig. 1). All subjects experienced intermittent circular vection, the first epoch after a mean latency of $9 \mathrm{~s}$ ( $\pm 4 \mathrm{~s}$ SD) after onset of disk rotation and ensuing epochs of perceived self- and object-motion with mean durations of 16 and 19s, respectively. Object- and self-motion percepts were mutually exclusive; i.e., during vection the disk was perceived as stationary and when disk rotation was perceived there was no simultaneous impression of self-motion. None of the subjects suffered from nausea. Subjects were familiarized with the stimulus and the percepts that it evokes and with the task prior to the experimental runs during functional imaging.

\section{Data Analysis}

Data processing and analysis used statistical parametric mapping (http://www.fil.ion.ud.ac.uk/spm). After discarding the initial eight, all image volumes were three-dimensionally realigned to the first volume, coregistered with the subject's corresponding anatomical (T1-weighted) images, nonlinearly normalized into standard stereotactic space (template provided courtesy of the Montreal Neurological Institute), and smoothed using a 9-mm full-width at half maximum Gaussian kernel. We removed low-frequency fluctuations by a temporal high-pass filter with a cutoff time constant at $70 \mathrm{~s}$ and analyzed two types of responses, sustained (perceptual state) and transient (perceptual switch). These were modeled as boxcar functions (sustained) and delta functions (transient) that were convolved with a synthetic hemodynamic response function. Durations of states (percepts) and timing of events (switches) were derived from the subjects' key presses (see above).

We analyzed neural activity changes in relation to stimulation as a first and perception as a second level of experimental variable. Statistical comparisons were performed by contrasting parameter estimates for the modeled sustained responses during visual-motion stimulation (across both percepts) with the stationary baseline and, as an embedded comparison within ongoing constant visual-motion stimulation, by contrasting those responses during perceived object-motion with those during perceived self-motion and vice versa (see Fig. 1). For event-related responses, we analyzed the evoked transient hemodynamic responses, i.e., the events at real-time resolution convolved with a canonical hemodynamic response function, both as changes against basel ine activity and against each other (Kleinschmidt et al., 1998). The three event types reported by key presses (see above) were the onset of visual-motion, perceived self-motion, and perceived object-motion.

These procedures resulted in statistical parametric maps for every voxel showing sustained or transient activity differences related to the stimulus and to either of the percepts. Statistical inferences were corrected for multiple nonindependent comparisons by us- 
ing Gaussian random field theory. Unless stated otherwise we applied a significance threshold of $\mathrm{P}<$ 0.05 , corrected.

\section{Cortical Localization}

We assigned tentative area labels to group analysis response foci that were in part supported by functional responses determined here, i.e., sensitivity to optical flow (Figs. 2 and 3), or by stereotactic coordinates for localized functional response properties reported in the literature. In particular, we related activations in calcarine cortex to $\mathrm{V} 1$, in the posterior fusiform gyrus to V4, in the lateral occipito-temporal cortex to V5/MT, and in the posterior insula to vestibular cortex. The joint label of $\mathrm{V} 3 \mathrm{~N} 3 \mathrm{a}$ reflects that these areas are rather thin stripes and variable in position even in relation to sulcal landmarks. We chose the anatomically descriptive labels of dorso-medial cortex (DM) and superior temporal cortex (ST) to avoid implying a single visual area and merely conclude that the functional behavior in these foci suggests homologies with macaque areas V6/PO and STPa, respectively. Finally, the putative identification of area V5a/MST and PIVC is discussed below.

\section{RESULTS}

\section{Regions Responsive to the Visual-Motion Stimulus}

We determined responsiveness to the visual-motion stimulus (coherent wide-field stimulation) by contrasting activity between the period of rotation and the preceding stationary phase (see Fig. 1 and Materials and Methods). The spatial distribution of regional activations found here largely conforms with previous neuroimaging studies mapping the responses to visualmotion (Watson et al., 1993; Zeki et al., 1993; Dupont et al., 1994; de J ong et al., 1994; Tootell et al., 1995, 1997; Cheng et al., 1995; McCarthy et al., 1995; McKeefry et al., 1997, Dieterich et al., 1998; Greenlee, 2000; Previc et al., 2000). Since the response to the stimulus per se was not the effect of interest in this study we present only an overview of the cortical response topography (Fig. 2).

\section{Regions Responsive to Perceptual State}

The experimental parameter of interest in our study was that, over time, all subjects perceived object-motion and circular vection in irregular alternation (Fig. 1). Reports of these perceptual switches enabled us to assign our data to one or other percept (see Materials and Methods). Hence, we determined activity levels during the two percepts in motion-sensitive candidate regions that animal electrophysiology, human lesion studies (Straube and Brandt, 1987; Heide et al., 1990; Vaina, 1998), and neuroimaging studies had suggested as important for optical flow processing. This included
A visual-motion (stimulation)
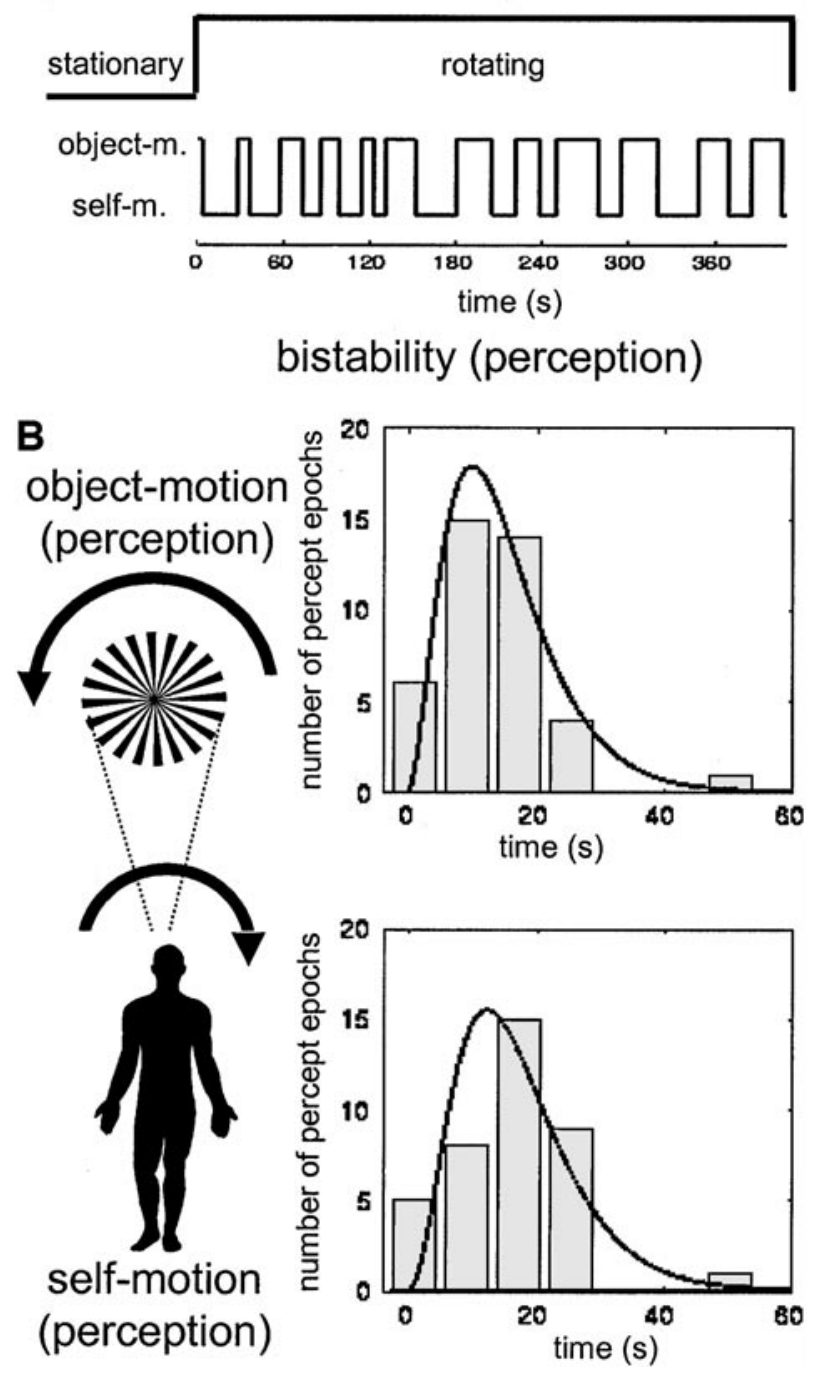

FIG. 1. Experimental design and perceptual behavior. (A) After an initial baseline period, a stationary disk with a windmill pattern starts rotating. While fixating its center of rotation, subjects switch between the percept of object-motion and that of self-motion, i.e., circular vection. Data obtained while scanning one of the participating subjects are shown as a time course alternating between the two percepts of object-motion and self-motion (vection). (B) Distribution of durations for each percept for the same data as shown in A. The epoch length histograms were approximated by gamma functions (bin width 8.2 s, i.e., equivalent to two sampled image volumes), a temporal switch distribution typical of bistable perception (Kleinschmidt et al., 1998; Sterzer et al., 2002).

DM (comprising cuneus and parieto-occipital cortex; see Richer et al. (1991) and Lee et al. (2000) for electrostimulation studies), the anterior portion (putative "V5a/MST") of the human motion complex ("V5/MT complex") at the occipito-temporal junction (Duffy and Wurtz, 1991; Orban, 1997; Tanaka, 1998; Wurtz, 1998), an area of ST (see Bruce et al., 1981; Anderson and Siegel, 1999), and posterior (intra-) parietal cortex (see Siegel and Read, 1997). All these regions re- 


\section{visual-motion > stationary}

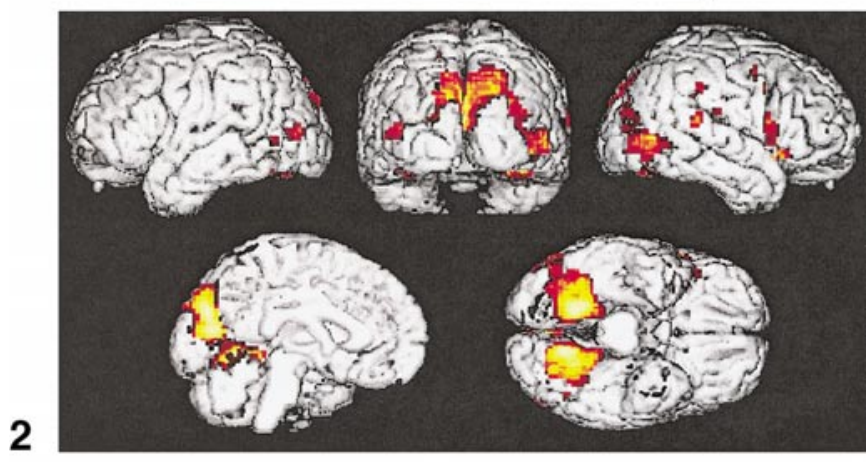

\section{object-motion $=$ self-motion}

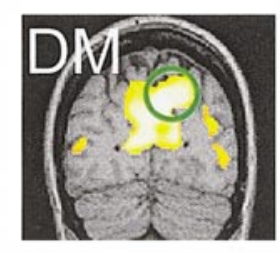

3
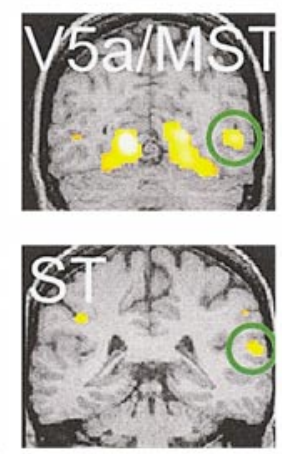

FIG. 2. Brain areas responsive to optokinetic visual-motion. Statistical parametric maps showing areas with greater activity during visual-motion (disk rotation) than during the preceding baseline (disk stationary). Toillustrate the overall cortical response pattern in a group analysis (three repetitions per subject of the stimulation protocol shown in Fig. 1A), the results are displayed by color-coding and rendered onto an anatomical template image (visualized at $P<0.05$, corrected).

FIG. 3. Motion-sensitive brain areas equally responsive during perceived object-motion and self-motion. Statistical parametric maps (left) from a group analysis of areas responsive to visual-motion (disk rotation) compared to baseline (disk stationary). These maps are derived from the same statistical comparison as in Fig. 2, color-coded, and superimposed onto sections from an individual structural dataset of a participating subject (visualised at $\mathrm{P}<0.001$, uncorrected). The location of the significance maxima contained in green circles is given in terms of stereotactic coordinates and with tentative area labels (see Cortical Localization in Materials and Methods; DM, dorsomedial cortex; ST, superior temporal area; V5a/MST, accessory V5/medial superior temporal area). The graphs on the right plot response strength in these foci (in the circled maximally significant responsive voxels at the coordinates given) for perceived object-motion and self-motion relative to the stationary phase (parameter estimates of percentage signal change averaged across runs and subjects; note differences in y axis scaling). There was no significant difference in activity levels across the two percepts ( $P<0.001$, uncorrected). Black vertical bars indicate standard error of the mean, i.e., topographical and functional intersubject variability, and are not related to response identification (which is represented by the statistical parametrical maps).

\section{object-motion > self-motion}
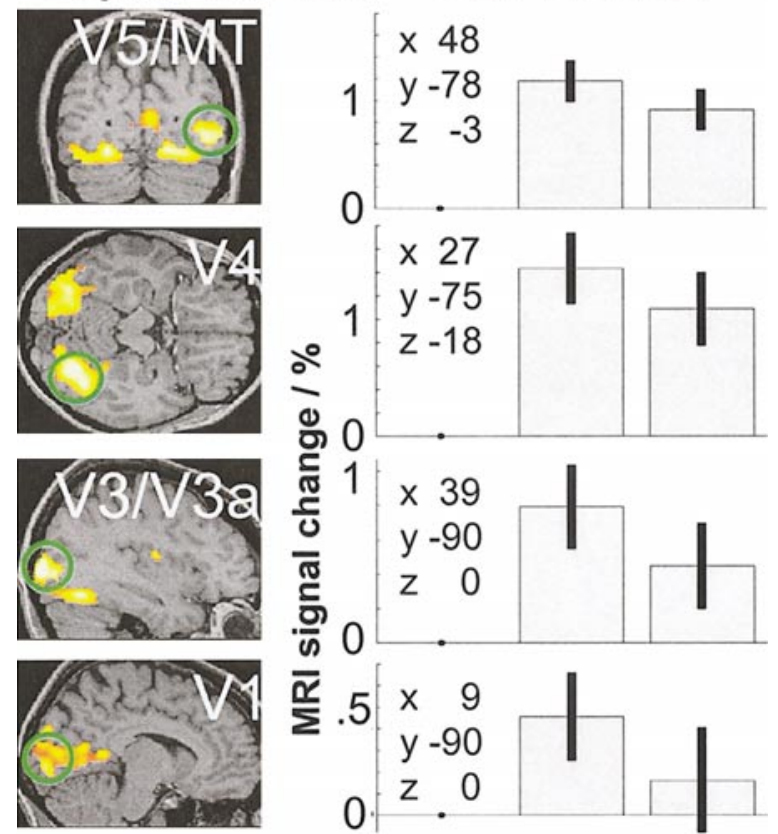

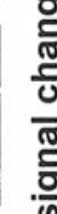
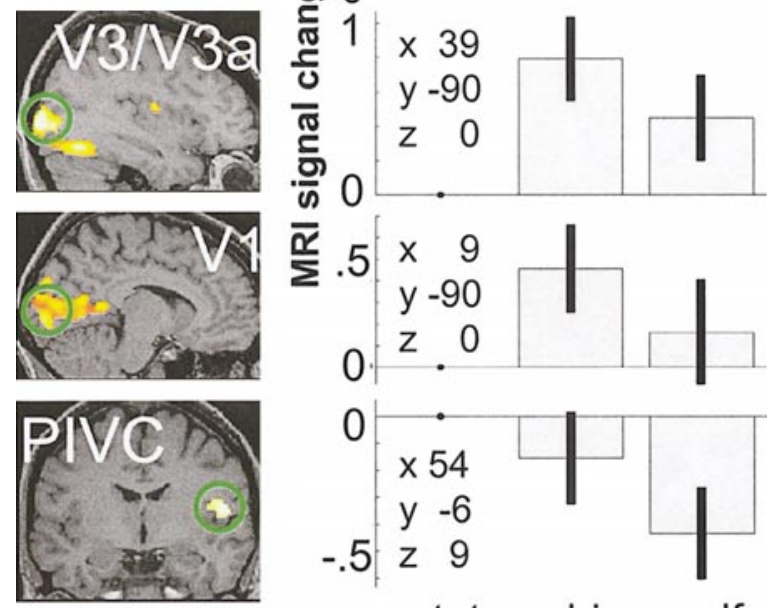

stat. obj. self

FIG. 4. Brain areas activated during perceptual dominance of object-motion. The statistical parametric maps on the left were obtained from a group analysis testing for greater activity during perceived object-motion than during circular vection (visualised at $\mathrm{P}<0.001$, uncorrected and superimposed onto individual structural sections). Response foci are highlighted by green circles and were tentatively labeled (cf. Fig. 3; V1-V5, visual areas 1-5; PIVC, parieto-insular vestibular cortex). Correspondingly, activity levels in the maximally percept-sensitive voxels in these foci (coordinates inserted) are plotted in the adjacent panels for each percept relative to baseline. Note differences in y axis scaling for the plots. All differences shown were significant at the level of $\mathrm{P}<0.05$, corrected.

sponded to our stimulus compared to the stationary baseline (see Fig. 2) but showed no differential activation in relation to the two different perceptual states (Fig. 3). For these comparisons, we used a more sensitive threshold ( $P<0.001$, uncorrected) that took account of the greatly reduced number of multiple comparisons involved and analyzed single subjects. This means that our negative result was not accounted for by overly conservative statistical thresholds or by blurred localization due to group analysis.

In the next step, we tested our data for an influence of perceptual state on regional activity levels. This was done by mapping activity differences between images reflecting the percept of object-motion and those reflecting the percept of self-motion and vice versa. When contrasting perceived object- with self-motion (Fig. 4), activity in a subset of the motion-sensitive brain areas 


\section{self-motion > object-motion}
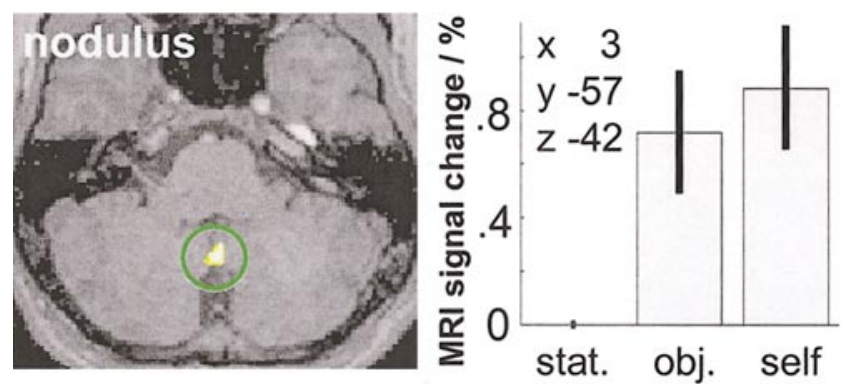

FIG. 5. Theresponse focus for perceptual dominance of self-motion. Statistical parametric map from a group analysis testing for greater activity during perceived self-motion than object-motion showing the cerebellar nodulus and plot of the associated parameter estimates (statistical comparison opposite to that in Fig. 4; same procedure, significance, and visualization thresholds). Note that all individual structural scans of the participating subjects were inspected at this location to rule out an underlying or adjacent large vascular structure that might form a potential nonspecific source of contrast change.

shown in Fig. 2 showed greater activity during perceived object-motion and less activity during perceived self-motion. The "earliest" percept-sensitive cortical ac- tivity change occurred in primary (calcarine) visual cortex ("VI") and extended over an intermediate level in the superior occipital gyrus ("V3N3A") into ventral occipital cortex (fusiform gyrus, "V4") and onto the convexity (occipito-temporal junction, posterior "V5/ MT"). These activity differences were significant in the group analysis even at thresholds corrected for multiple comparisons but, again, were not significant when analyzed in single subjects.

A significant activity difference also occurred in an area not activated by the visual-motion stimulus, namely, the posterior parieto-insular cortex, a region proposed to be a human homologue of a vestibular cortex (Fig. 4, bottom). Compared to activity levels during the stationary stimulus, this difference results from a deactivation during perceived self-motion. Hence, while activity levels were not significantly different between the stationary and the rotating stimuli as long as the latter was perceived as object-motion, there was a significant activity decrease during illusory self-motion.

In contrast to the large set of motion-sensitive areas with greater activity during perceived object-motion,

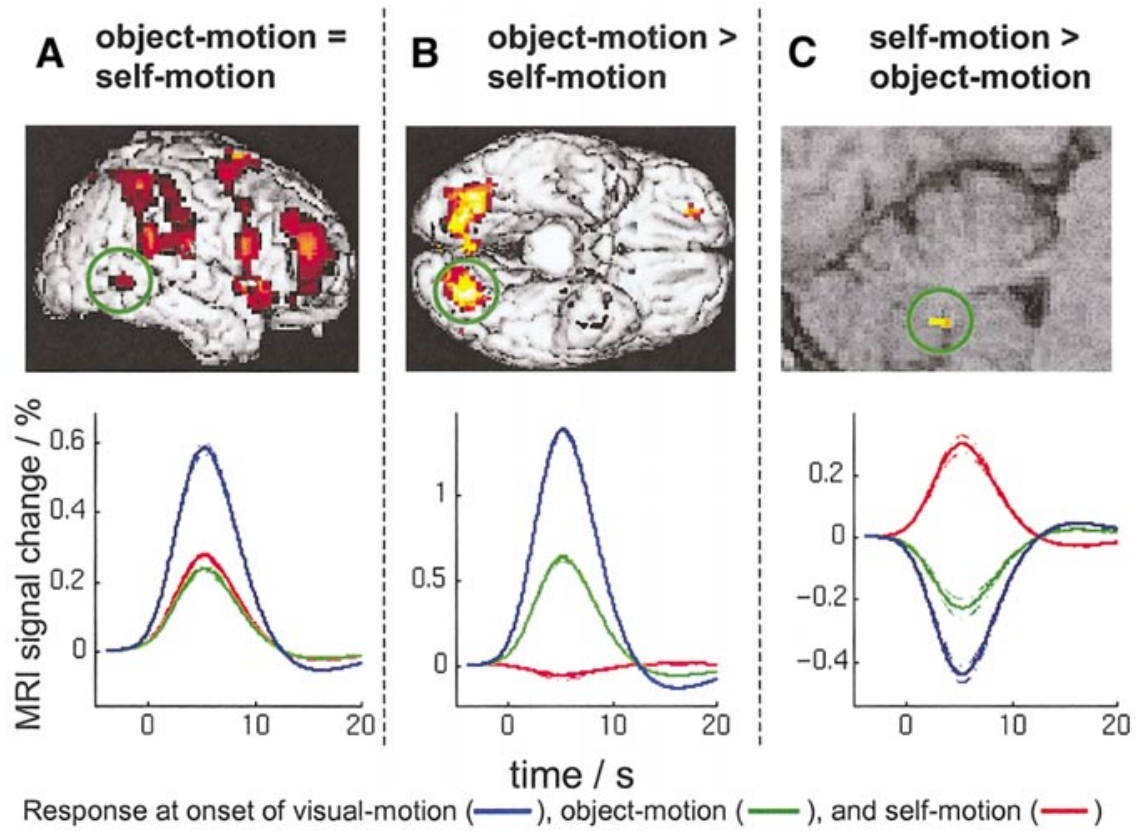

FIG. 6. Results from a group analysis of transient activations during perceptual reversals. (A) The regions responsive to both the perceived onset of self-motion and that of object-motion are shown by rendering them onto a surface reconstruction of the right hemisphere (regions responding to vection onset and to perceived object-motion onset, both thresholded at $\mathrm{P}<0.001$; the latter enacted by using an inclusive mask option). Note that unlike the motion-sensitive areas shown in Fig. 2 there is predominant activation in a fronto-parietal system, whereas effects in visual areas are restricted to dorsomedial parieto-occipital cortex (not shown) and the anterior pole of the temporo-occipital motion complex. The modeled responses (solid line) and their standard error (hatched) in the peak voxel from that latter area (green circle) are plotted below in response to the three event types at time 0 (for use of colors for event types see bottom). (B) The responses at the perceived onset of object-motion. The result of the comparison of greater responses during the onset of perceived object-motion than of self-motion (at $\mathrm{P}<0.001$ ) are displayed on a rendering of the ventral brain surface (cerebellum removed) with the underlying modeled responses (from peak voxel in the green circle) to the three event types plotted below (for colors see bottom). (C) The responses at the perceived onset of self-motion. The result of the comparison of greater responses during the onset of perceived self-motion than of object-motion (at $P<0.001$ ) is displayed on a medio-sagittal section of the structural brain scan of one of the participating subjects and the underlying modeled responses (solid line) for the three different event types are plotted below (for colors see bottom). 
only one structure showed enhanced activity during vection relative to perceived object-motion ( $F$ ig. 5), the nodulus in the cerebellar vermis (possibly extending into the adjacent uvula). The nodulus is a subcortical structure responsive to the optokinetic visual-motion stimulus compared to the stationary control. Other subcortical motion-sensitive responses occurred in the flocculus and thalamus but these showed no differential responsiveness as a function of perceptual state.

\section{Regions Responsive to Perceptual Change}

In event-related analyses, we localized responses to perceptual switching from seeing a rotating disk ("object-motion") to vection ("self-motion") and vice versa. Perceptual states lasted sufficiently long to decorrelate percept- and switch-related activity (Fig. 1). Accordingly, only event-related analyses showed activation in left-hemispheric hand motor areas on the convexity and medial wall related to the subjects' key presses at perceptual switches. Overall, the event-related re sponses that we found fell into three classes: those common to both directions of perceptual reversal (from perceived object-motion to self-motion and vice versa) and those specific to either of the two directions.

In line with previous observations (Kleinschmidt et al., 1998; Lumer et al., 1998; Sterzer et al., 2002), transient activations at perceptual switches, irrespective of their direction, occurred in predominantly righthemispheric inferior and intra-parietal cortex and premotor, inferior frontal, and prefrontal cortex. Similar activations were found in the anterior portion of the right-sided motion complex ("V5a/MST;" Fig. 6A). This means that the sustained response to visual-motion in that area was modulated by transient activations at each perceptual reversal that were of similar magnitude for perceived onset of object-motion and selfmotion. The same pattern was also found in the parieto-occipital region ("DM"), which showed identical sustained activity in either perceptual state, and, nonsignificantly, in the right superior temporal region.

We also observed transient activations that occurred only at the onset of perceived object-motion (Fig. 6B). This functional behavior was found in all early visualmotion-sensitive regions with greater sustained activity during perceived object-motion than during vection and is thus interpretable as the phasic component of the tonic response identified in state-related analyses. Similarly, a transient activation at the onset of perceived self-motion was found only in the cerebellar nodulus (Fig. 6C).

\section{DISCUSSION}

Vection as a Tool to Address Neural Correlates of Self-Motion Perception

Previous neuroimaging studies on the visual processing of self-motion have pursued the experimental avenue that self-motion inevitably generates complex but widely coherent visual-motion, so-called optical flow fields (Gibson, 1954). In these studies (de J ong et al., 1994; Brandt et al., 1998; Cheng et al., 1995; McKeefry et al., 1997; Greenlee, 2000; Previc et al., 2000), flow responses were compared to responses to visual stimuli that cannot originate from self-motion, thus identifying candidate areas for the perception of selfmotion by virtue of their sensory response properties. Here, we correlated brain activity changes with the percept of self-motion while controlling for the underlying visual-motion stimulus. We show that, during continuous constant visual-motion stimulation, motion-sensitive areas in lateral temporo-occipital ("MST/ V5a") and medial parieto-occipital cortex ("DM") do not change activity as a function of percept (object- or self-motion; Fig. 3) but transiently activate whenever perception switches (Fig. 6). Furthermore, all earlier motion-sensitive visual areas and the parieto-insular vestibular cortex deactivate during perceived self-motion (Fig. 4).

Self-motion perception in our experiment is illusory as functional neuroimaging currently precludes true observer-motion. However, this model to study visual processing during self-motion is valid because afferent input during vection imitates that from constant-vel ocity self-motion. A not actively moving, but steadily displaced observer (zero acceleration) will lack congruent proprioceptive and vestibular input, e.g., while driving a car at constant velocity and fixating the end of a straight road, and reconstruction of self-motion will hence exclusively depend on visual-motion input. As a consequence rather than a failure of these sensory mechanisms, an appropriate visual-motion stimulus can evoke the sensation of self-motion in a stationary observer. Adjusting our subjects' perceptual interpretation of such a stimulus to bistability, with the two alternating perceptual states lasting for roughly equivalent lengths of time, enabled us to compare brain activity while stimulation was constant but perception fluctuated between two different states, i.e., object- and self-motion.

\section{Functional Behavior of Temporo- and Parieto- Occipital Cortex during Vection}

Compared to stationarity the rotation of the windmill pattern evoked a widespread response pattern. Both its spatial distribution and the overall right hemispheric predominance (Fig. 2) are to be expected for this type of stimulus. Moreover, this pattern included the candidate regions for the visual processing of selfmotion (see below). Surprisingly, however, activity levels in these areas did not differ as a function of the dominant percept. This functional behavior was for instance found in the anterior portion (putative homologue of V5a/MST) but not the posterior portion of the lateral occipito-temporal motion complex. This obser- 
vation suggests a functional subdivision of the human motion complex, with the posterior portion putatively reflecting the functional behavior of area V5/MT and the anterior that of area V5a/MST(d). This interpretation is compatible with a recent fMRI study that compared the responses to circular and radial flow to those to translational visual-motion and identified a specifically flow-sensitive area within the human motion complex V5/MT as a putative human homologue of MSTd (Morrone et al., 2000). Stereotactic coordinates reported for that area relative to responses to translational motion were at the anterior (and ventral) end of the motion complex.

Electrophysiological recordings in the dorsal medial superior temporal area (MSTd) of nonhuman primates have shown preferential responses to flow stimuli (Duffy and Wurtz, 1991; Orban, 1997; Tanaka, 1998) and congruent vestibular input with real self-motion (Duffy, 1998; Bremmer et al., 1999). These neuronal response properties suggest that this area is ideally suited to contribute to the reconstruction of self-motion (Wurtz, 1998; Andersen et al., 2000) although other areas, which have been less well characterized by single-unit recordings, may also play an important role. Our findings support the notion that this area is not the only one relevant for self-motion perception be cause a similar functional behavior was found in several other areas including a dorsomedial parieto-occipital region (DM, putatively corresponding to area V6/ PO; see Galletti et al., 1999; Rosa and Tweedale, 2001) but also in superior and intra-parietal foci and superior temporal foci (putatively corresponding to the anterior superior temporal polysensory area described in nonhuman primates; see Bruce et al., 1981; Cusick, 1997; Anderson and Siegel, 1999).

The question with regard to these candidate areas, however, is whether identical (instead of increased) activity levels during perceived self-motion (as compared to object-motion) are compatible with a functional role of these areas in the perception of selfmotion. As a visual stimulus, self-motion is only one of the multiple cases of complex visual-motion. Any visual area implicated in self-motion processing will inevitably also display response properties suitable for other functional contexts, and processing of these contexts may interact (Probst et al., 1984; Geesaman and Andersen, 1996; Recanzone et al., 1997; Zemel and Sejnowski, 1998; but see also Royden and Hildreth, 1999). We therefore propose that the lateral temporooccipital ("MSTN5a") and medial parieto-occipital areas ("DM"), where activity remained constantly elevated during both percepts, process the visual-motion input arising from self-motion but also other types of coherent visual-motion.

This interpretation is compatible with the sustained responses that we found in the candidate areas for self-motion perception. However, an area that is sensitive to the perceptual consequence of a visual stimu-
Ius should also display transient signal changes whenever the perceptual interpretation changes. Using ambiguous visual stimuli that maintain a constant sensory input, event-related activations during perceptual switches have been found in several extrastriate areas and their localization conforms with the sensory response properties of those areas. Hence, perceptual changes related to objects and faces were accompanied by fusiform activations (Kleinschmidt et al., 1998) and changes related to motion direction by activations in temporo-occipital visual-motion areas (Sterzer et al., 2002).

Our event-related analyses here show that, over and above a continuous response to the visual-motion stimulus, lateral temporo-occipital ("MSTN5a") and medial parieto-occipital areas ("DM") activated transiently each time perception changed from one possible interpretation to the other. Different from other areas, these responses were equivalent for both directions of perceptual change, as were the sustained activity levels for both percepts. Interestingly, in the aforementioned study by Morrone et al. (2000), the best responses in putative MSTN5a were elicited by changes in optic flow direction rather than constant flow which is also in line with electrophysiological findings in MST (Paolini et al., 2000; see also Duffy and Wurtz, 1997). We also observed transient activations in other brain areas that seem related to more general aspects of perceptual synthesis and selection (Kleinschmidt et al., 1998; Lumer et al., 1998; Sterzer et al., 2002) and activations during only one direction of perceptual change that occurred in regions sensitive to one type of percept but not the other, as discussed in the following section.

Brain Activity Changes Related to the Percept of Object- or Self-Motion

Comparing sustained activity levels in the two perceptual states, we found greater activity during perceived object-motion in a set of areas reaching from cal carine cortex ("V1") to ventral occipital cortex ("V4") and lateral temporo-occipital cortex ("V5"). The latter focus corresponds to the posterior portion of the motion complex; the former was in the fusiform gyrus. These two functional ramifications of the visual system are differentially engaged by color, form, and motion, and their activation during perceived object-motion might indicate integrative segregation by construction of "form-from-motion" (Lamme et al., 1993; Van Essen and Gallant, 1994).

The alternative interpretation for differential activity in the two percepts is a deactivation in early motion-sensitive areas during vection. We had no eyemovement recording facilities available in this study but the optokinetic stimulus that we used reliably elicits a torsional nystagmus (Brecher, 1934; Finke and Held, 1978; Cheung and Howard, 1991) which fails 
almost completely to cancel retinal slip. Its gain (ratio between stimulus velocity and eye velocity) is $\sim 0.1$ but increases by $\sim 40 \%$, from 0.08 to 0.12 , during vection (Thilo et al., 1999). For a stimulus of $45 \%$ s this translates into less than $2 \% \mathrm{~s}$ retinal stimulation difference between the two percepts-too little to account for the profound activity difference found in multiple visual areas.

The influence of extraretinal signals related to the small but significant changes in the slow and fast (saccadic) components of nystagmus during self-motion perception is more difficult to evaluate. Saccades (and vestibularly elicited saccades) suppress activity in early visual cortex (Paus et al., 1995; Wenzel et al., 1996; Gallant et al., 1998) but in extrastriate visual areas of the parvo-dominated ventral stream perisaccadic modulation either is absent or is even excitatory (Leopold and Logothetis, 1998). Similarly, the modulatory influence of slow-pursuit eye movements on activity in the human motion complex seems to be, if anything, excitatory (Freitag et al., 1998). It therefore appears unlikely that the widespread deactivations in several visual areas (and lack of activation in candidate self-motion perception areas) during vection merely reflect a slight percept-dependent gain change of the associated nystagmus.

We did find enhanced activity during vection in the cerebellar nodulus (and maybe the adjacent uvula). The nodulus is a critical site both for visuo-vestibular interaction (Precht et al., 1976) and for torsional optokinetic nystagmus (Angelaki and Hess, 1994). Nodulus activation during vection could therefore be accounted for by the oculomotor gain increase or by changes in sensory processing related to the rotational percept (reviewed in Cohen et al., 1999). Y et, nystagmus facilitation is only one of several functionally plausible neuronal adjustments to self-motion. In fact, deactivations in early motion-sensitive areas, nystagmus facilitation, and saccadic suppression may all share a common functional purpose. Greater nystagmus gain during observer-motion decreases retinal slippage of the environment and perisaccadic suppression of re lated retinal slip stabilizes visual perception against the effects of eye movements. Yet, a functional benefit from suppressing observer-induced retinal slip would also apply to head and body movements (Crowell et al., 1998). A more general suppressive mechanism during self-motion could thus ensure that despite retinal slippage due to eye, head, and body movements, ongoing visual processing remains undisturbed (Probst et al., 1984; Wexler et al., 2001). During self-motion such a suppression would prove helpful only if it selectively spares those visual cortical areas specialized for the flow fields arising from self-motion. This is the case in areas that maintain a veridical visual-motion activity profile across both percepts (Fig. 3). Even though they do not activate further during vection (compared to object-motion) they nevertheless remain activated
(Fig. 3) as opposed to the deactivation in earlier motion-sensitive areas (Fig. 4).

\section{Visuo-Vestibular Interactions during Vection}

In addition to perceptually driven visuo-visual interactions (between cortices processing object-motion and self-motion) we found corresponding visuo-vestibular interactions. It was shown previously that a parietoinsular region identified as a human "vestibular" cortex (Bottini et al., 1994; see also Guldin and Grüsser, 1998) is deactivated by visual-motion stimuli that generate vection (Brandt et al., 1998). The critical finding in our study is that this deactivation results not from the stimulus per se but from its perceptual interpretation (Fig. 4, bottom). Hence, during a constant visualmotion stimulus, the percept of self-motion is accompanied by deactivations of structures that process object-motion and vestibular information, i.e., potential distracters to the visual analysis of steady selfmotion (Brandt, 1999). In accordance with this view, the neural signature of self-motion perception is not the activity of a single "self-motion center" but a distributed activity pattern of interconnected areas that process spatial sensory cues such as retinal and vestibular input.

\section{CONCLUSION}

Our study addressed how the human brain processes two alternative perceptual results of an ambiguous visual-motion stimulation that differ profoundly in functional meaning. The illusion of self-motion, intermittently generated by our stimulus, provides a model of how the brain deals with retinal stimulation that results from our own actions. While there is a general suppression of activity in visual and vestibular cortex during vection, the activity levels in advanced visual processing stages remain unaffected and thus presumably allow for continued reconstruction of self-motion. The effects that we observed can be functionally framed as mechanisms that allow us to stabilize our perception of the visual environment and to use our visual sense to guide our locomotion.

\section{ACKNOWLEDG MENTS}

We thank Mr. William Cameron from the MRC for constructing the stimulus setup and the unit's physics and methods teams for continuous support. A.K. and C.B. are supported by the VolkswagenStiftung, K.V.T by the Dix Foundation, M.A.G. and A.M.B. by the Medical Research Council, and R.S.J .F. by the Wellcome Trust.

\section{REFERENCES}

Albright, T. D., and Stoner, G. R. 1995. Visual-motion perception. Proc. Natl. Acad. Sci. USA 92: 2433-2440.

Andersen, R. A., Shenoy, K. V., Crowell, J. A., and Bradley, D. C. 2000. Neural mechanisms for self-motion perception in area MST. Int. Rev. Neurobiol. 44: 219-233. 
Anderson, K. C., and Siegel, R. M. 1999. Optic flow sensitivity in the anterior superior temporal polysensory area, STPa, of the behaving monkey. J. Neurosci. 19: 2681-2692.

Angelaki, D. E., and Hess, B. J . M. 1994. The cerebellar nodulus and ventral uvula control the torsional vestibuloocular reflex. J . Neurophysiol. 72: 1443-1447.

Bottini, G., Sterzi, R., Paulesu, E., Vallar, G., Cappa, S. F., Erminio, F., Passingham, R. E., Frith, C. D., and Frackowiak, R. S. F. 1994. Identification of the central vestibular projections in man: A positron emission tomography activation study. Exp. Brain Res. 99: $164-169$.

Brandt, T. 1999. Cortical visual-vestibular interaction for spatial orientation and self-motion perception. Curr. Opin. Neurol. 12: 1-4.

Brandt, T., Bartenstein, P., J anek, A., and Dieterich, M. 1998. Reciprocal inhibitory visual-vestibular interaction: Visual motion stimulation deactivates the parieto-insular vestibular cortex. Brain 121: 1749-1758.

Brecher, G. A. 1934. Die optokinetische Auslösung von Augenrollung und rotatorischem Nystagmus. Pflüg. Arch. Ges. Physiol. 234: 13-28.

Bremmer, F., Kubischik, M., Pekel, M., Lappe, M., and Hoffmann, K.P. 1999. Linear vestibular self-motion signals in monkey medial superior temporal area. Ann. N. Y. Acad. Sci. 871: 272-281.

Bruce, C. J ., Desimone, R., and Gross, C. G. 1981. Visual properties of neurons in a polysensory area in superior temporal sulcus of the macaque. J . Neurophysiol. 46: 369-384.

Cheng, K., Fujita, H., Kanno, I., Miura, S., and Tanaka, K. 1995. Human cortical regions activated by wide-field visual motion: An H2150 PET study. J . Neurophysiol. 74: 413- 427.

Cheung, B. S., and Howard, I .P. 1991. Optokinetic torsion: Dynamics and relation to circularvection. Vis. Res. 31: 1327-1335.

Cohen, B., Wearne, S., Dai, M., and Raphan, T. 1999. Spatial orientation of the angular vestibulo-ocular reflex. J . Vestib. Res. 9: 163-172.

Crowell, J . A., Banks, M. S., Shenoy, K. V., and Andersen, R. A. 1998. Visual self-motion perception during head turns. Nat. Neurosci. 1: 732-737.

Cusick, C. G. 1997. The superior temporal polysensory region in monkeys. In Cerebral Cortex, Vol. 12: Extrastriate Cortex (K. S. Rockland, J . H. Kaas, and A. Peters, Eds.), pp. 435- 468. Plenum, New York.

de J ong, B. M., Shipp, S., Skidmore, B., Frackowiak, R. S. J ., and Zeki, S. 1994. The cerebral activity related to the visual perception of forward motion in depth. Brain 117: 1039-1054.

Dichgans, J., and Brandt, T. 1978. Visual-vestibular interaction: Effects on self-motion perception and postural control. In Handbook of Sensory Physiology (R. Held, H. W. Leibowitz, and H. L. Teuber, Eds.), Vol. 8, pp. 755- 804. Springer-Verlag, Heidelberg.

Dieterich, M., Bucher, S. F., Seelos, K. C., and Brandt, T. 1998. Horizontal or vertical optokinetic stimulation activates visual motion-sensitive, ocular motor and vestibular cortex areas with right hemispheric dominance. Brain 121: 1479-1495.

Duffy, C. J . 1998. MST neurons respond to optic flow and translational movement. J . Neurophysiol. 80: 1816-1827.

Duffy, C. J ., and Wurtz, R. H. 1991. Sensitivity of MST neurons to optic flow stimuli. I. A continuum of response selectivity to largefield stimuli. J . Neurophysiol. 65: 1329-1345.

Duffy, C. J ., and Wurtz, R. H. 1997. Multipletemporal components of optic flow responses in MST neurons. Exp. Brain Res. 114: 472482.

Dupont, P., Orban, G. A., de Bruyn, B., Verbruggen, A., and Mortelmans, L. 1994. Many areas in the human brain respond to visual motion. J . Neurophysiol. 72: 1420-1424.
Finke, R. A., and Held, R. 1978. State reversals of optically induced tilt and torsional eye movements. Percept. Psychophys. 23: 337340.

Freitag, P., Greenlee, M. W., Lacina, T., Scheffler, K., and Radu, E. W. 1998. Effect of eye movements on the magnitude of functional magnetic resonance imaging responses in extrastriate cortex during visual motion perception. Exp. Brain Res. 119: 409414.

Gallant, J. L., Connor, C. E., and Van Essen, D. C. 1998. Neural activity in areas V1, V2 and V4 during free viewing of natural scenes compared to controlled viewing. NeuroReport 9: 1673-1678.

Galletti, C., Fattori, P., Gamberini, M., and Kutz, D. F. 1999. The cortical visual area V6: Brain location and visual topography. Eur. J . Neurosci. 11: 3922-3936.

Geesaman, B. J ., and Andersen, R. A. 1996. The analysis of complex motion patterns by form/cue inavariant MSTd neurons. J . Neurosci. 16: 4716- 4732.

Gibson, J . J . 1954. The visual perception of objective motion and subjective movement. Psychol. Rev. 61: 304-314.

Greenlee, M. W. 2000. Human cortical areas underlying the perception of optic flow: Brain imaging studies. Int. Rev. Neurobiol. 44: 269-292.

Guldin, W. O., and Grüsser, O. J . 1998. Is there a vestibular cortex? Trends Neurosci. 21: 254-259.

Heide, W., Koenig, E., and Dichgans, J. 1990. Optokinetic nystagmus, self-motion sensation and their aftereffects in patients with occipito-parietal lesions. Clin. Vis. Sci. 5: 145-156.

Helmholtz, H. 1896. Handbuch der Physiologischen Optik. Voss, Leipzig.

Kleinschmidt, A., Büchel, C., Zeki, S., and Frackowiak, R. S. J . 1998. Human brain activity during spontaneously reversing perception of ambiguous pictures. Proc. R. Soc. Lond. B 265: 2427-2433.

Lamme, V. A. F., Vandijk, B. W., and Spekreijse, H. 1993. Contour from motion processing occurs in primary visual cortex. Nature 363: 541-543.

Lappe, M., Bremmer, F., and van den Berg, A. V. 1999. Perception of self-motion from visual flow. Trends Cogn. Sci. 3: 329-336.

Lee, H. W., Hong, S. B., Seo, D. W., Tae, W. S., and Hong, S. C. 2000. Mapping of functional organization in human visual cortex: Electrical cortical stimulation. Neurology 54: 849-854.

Leopold, D. A., and Logothetis, N. K. 1998. Microsaccades differentially modulate neural activity in the striate and extrastriate visual cortex. Exp. Brain Res. 123: 341-345.

Lumer, E. D., Friston, K. J ., and Rees, G. 1998. Neural correlates of perceptual rivalry in the human brain. Science 280: 1930-1934.

Mach, E. 1875. Grundlinie der Lehrevon den Bewegungsempfindungen. Engelmann, Leipzig.

McCarthy, G., Spicer, M., Adrignolo, A., Luby, M., Gore, J., and Allison, T. 1995. Brain activation associated with visual motion studied by functional magnetic resonance imaging in humans. Hum. Brain Mapp. 2: 234-243.

McKeefry, D. J ., Watson, J . D. G., Frackowiak, R. S. J ., and Zeki, S. 1997. The activity in human areas V1/N2, V3, and V5 during the perception of coherent and incoherent motion. Neurolmage 5: $1-12$.

Morrone, M. C., Tosetti, M., Montanaro, D., Fiorentini, A., Cioni, G., and Burr, D. C. 2000. A cortical area that responds specifically to optic flow, revealed by fMRI. Nat. Neurosci. 3: 1322-1328.

Movshon, J. A., Adelson, E. H., Gizzi, M. S., and Newsome, W. T. 1985. The analysis of moving visual patterns. Exp. Brain Res. Suppl. 11: 117-151.

Nakayama, K. 1985. Biological image motion processing. Vision Res. 25: 625- 660 .

Orban, G. A. 1997. Visual processing in macaque area MTN5 and its satellites (MSTd and MSTv). In Cerebral Cortex, Vol. 12: Extra- 
striate Cortex (K. S. Rockland, J . H. Kaas, and A. Peters, Eds.), pp. 359- 434. Plenum, New York.

Paolini, M., Distler, C., Bremmer, F., Lappe, M., and Hoffmann, K. P. 2000. Responses to continuously changing optic flow in area MST. J . Neurophysiol. 84: 730-743.

Paus, T., Marrett, S., Worsley, K. J ., and Evans, A. C. 1995. Extraretinal modulation of cerebral blood flow in the human visual cortex-I mplications for saccadic suppression. J . Neurophysiol . 74: 2179-2183.

Precht, W., Simpson, J . I., and Llinas, R. 1976. Responses of Purkinje cells in rabbit nodulus and uvula to natural vestibular and visual stimuli. Pflügers Arch. 367: 1- 6.

Previc, F. H., Liotti, M., Blakemore, C., Beer, J ., and Fox, P. 2000. Functional imaging of brain areas involved in the processing of coherent and incoherent wide field-of-view visual motion. Exp. Brain Res. 131: 393- 405.

Probst, T., Krafczyk, S., Brandt, T., and Wist, E. R. 1984. Interaction between perceived self-motion and object-motion impairs vehicle guidance. Science 225: 536-538.

Recanzone, G. H., Wurtz, R. H., and Schwarz, U. 1997. Responses of MT and MST neurons to one and two moving objects in the receptive field. J . Neurophysiol. 78: 2904-2915.

Richer, F., Martinez, M., Cohen, H., and Saint-Hilaire, J . M. 1991. Visual-motion perception from stimulation of the human medial parietooccipital cortex. Exp. Brain Res. 87: 649-652.

Rosa, M. G. P., and Tweedale, R. 2001. The dorsomedial visual areas in new world and old world monkeys: Homology and function. Eur. J. Neurosci. 13: 421- 427.

Royden, C. S., and Hildreth, E. C. 1999. Differential effects of shared attention on perception of heading and 3-D object motion. Percept. Psychophys. 61: 120-133.

Siegel, R. M., and Read, H. L. 1997. Analysis of optic flow in the monkey parietal area 7a. Cereb. Cortex 7: 327-346.

Statistical parametric mapping [Online]. Wellcome Department of Cognitive Neurology, University College London, U.K. http://www. fil.ion.ucl.ac.uk/spm.

Sterzer, P., Russ, M. O., Preibisch, C., and Kleinschmidt, A. 2002. Neural correlates of spontaneous direction reversals in ambiguous apparent visual-motion. Neurol mage 15: 908-916.

Straube, A., and Brandt, T. 1987. Importance of the visual and vestibular cortex for self-motion perception in man (circular vection). Hum. Neurobiol. 6: 211-218.
Tanaka, K. 1998. Representation of visual motion in the extrastriate visual cortex. In High-Level Motion Processing (T. Watanabe, Ed.), pp. 295-313. MIT Press, Cambridge, MA.

Thilo, K. V., Probst, T., Bronstein, A. M., Ito, Y., and Gresty, M. A. 1999. Torsional eye movements are facilitated during perception of self-motion. Exp. Brain Res. 126: 495-500.

Tootell, R. B. H., Reppas, J . B., Kwong, K. K., Malach, R., Born, R. T., Brady, T. J ., Rosen, B. R., and Belliveau, J . W. 1995. Functional analysis of human MT and related visual cortical areas using magnetic resonance imaging. J . Neurosci. 15: 3215-3230.

Tootell, R. B. H., Mendola, J . D., Hadjikhani, N. K., Ledden, P. J ., Liu, A. K., Reppas, J. B., Sereno, M. I., and Dale, A. M. 1997. Functional analysis of V3A and related areas in human visual cortex. J . Neurosci. 17: 7060-7078.

Vaina, L. M. 1998. Complex motion perception and its deficits. Curr. Opin. Neurobiol. 8: 494-502.

Van Essen, D. C., and Gallant, J. L. 1994. Neural mechanisms of form and motion processing in the primate visual system. Neuron 13: 1-10.

Warren, W. H. 1995. Self-Motion: Visual Perception and Visual Control. Academic Press, New York.

Watson, J. D. G., Myers, R., Frackowiak, R. S. J., Hajnal, J . V., Woods, R. P., Mazziotta, J . C., Shipp, S., and Zeki, S. 1993. Area V5 of the human brain: Evidence from a combined study using positron emission tomography and magnetic resonance imaging. Cereb. Cortex 3: 79-94.

Wenzel, R., Bartenstein, P., Dieterich, M., Danek, A., Weindl, A., Minoshima, S., Ziegler, S., Schwaiger, M., and Brandt, T. 1996. Deactivation of human visual cortex during involuntary ocular oscillations-A PET activation study. Brain 119: 101-110.

Wertheim, A. 1994. Motion perception during self-motion-The direct versus inferential controversy revisited. Behav. Brain Sci. 17: 293-311.

Wexler, M., Panerai, F., Lamouret, I., and Droulez, J. 2001. Selfmotion and the perception of stationary objects. Nature 409: 8588.

Wurtz, R. H. 1998. Optic flow: A brain region devoted to optic flow analysis. Curr. Biol. 8: R554-R556.

Zeki, S., Watson, J . D., and Frackowiak, R. S. J . 1993. Going beyond the information given: The relation of illusory visual motion to brain activity. Proc. R. Soc. Lond. B 252: 215-222.

Zemel, R. S., and Sejnowski, T. J . 1998. A model for encoding multiple object motions and self-motion in area MST of primate visual cortex. J . Neurosci. 18: 531-547. 